\title{
Extended finite element methods for optimal control problems governed by Poisson equation in non-convex domains *
}

\author{
Tao Wang ${ }^{\dagger}$ Chao Chao Yang $\stackrel{\ddagger}{\dagger}$ Xiaoping Xie ${ }^{\S}$
}

\begin{abstract}
This paper analyzes two eXtended finite element methods (XFEMs) for linear quadratic optimal control problems governed by Poisson equation in non-convex domains. We follow the variational discretization concept to discretize the continuous problems, and apply an XFEM with a cut-off function and a classic XFEM with a fixed enrichment area to discretize the state and co-state equations. Optimal error estimates are derived for the state, co-state and control. Numerical results confirm our theoretical results.
\end{abstract}

Keywords: extended finite element method, optimal control, non-convex domain, variational discretization concept.

\section{Introduction}

We consider the following linear quadratic optimal control problem:

$$
\min J(y, u):=\frac{1}{2} \int_{\Omega}\left(y-y_{d}\right)^{2} d x+\frac{\alpha}{2} \int_{\Omega} u^{2} d x
$$

for $(y, u) \in H_{0}^{1}(\Omega) \times L^{2}(\Omega)$ subject to Poisson equation

$$
\begin{cases}-\Delta y=u+f & \text { in } \Omega, \\ y=0 & \text { on } \partial \Omega,\end{cases}
$$

with the control constraint

$$
u_{0} \leq u \leq u_{1} \text {, a.e. on } \Omega \text {, }
$$

where $\Omega$ is a bounded polygonal domain in $\mathbb{R}^{2}$ with a single re-entrant corner of angle $\frac{\pi}{\beta}, \beta \in\left[\frac{1}{2}, 1\right] . y_{d} \in$ $L^{2}(\Omega)$ is the desired state to be achieved by controlling $u$. $\alpha$ is a positive constant and $f, u_{0}, u_{1} \in L^{2}(\Omega)$ with $u_{0} \leq u_{1}$ a.e. on $\Omega$. For the sake of simplicity, we choose homogeneous boundary condition on $\partial \Omega$. In fact, we can obtain similar results for other boundary conditions.

For the Poisson problem (1.2) in non-convex domains, it is well-known that the weak solution $y$ is generally not in $H^{2}(\Omega)$, due to the singularities at the corner. The low regularity may lead to reduced accuracy for finite element approximations [9]. In literature there are two ways to improve the accuracy. The first way is to use graded meshes (cf. [29, 6, 34, 3]). The second way is to use some singular basis functions which characterize the singularity of the solution around the corner; see, for instance, the classic singular enrichment method [39], the dual singular function method [15], and the singular complement method [23]. Notice that when $\Omega$ is a crack domain $\left(\beta=\frac{1}{2}\right)$, all the above methods have to use bodyfitted meshes. However, it is often difficult or expensive to construct such kinds of meshes, especially in time dependent problems.

In the past few decades, many numerical methods have been developed for the optimal control problem (1.1)-(1.3) in convex domains, see [16, 17, 42, 10, 25, 35, 13, 20, 32, 33, 31, 18, 25, 21, 22, 26, 44]. However, for optimal control problems in non-convex domains, there is only limited research work. In $[2,1,4]$, finite element error estimates were derived on graded meshes.

\footnotetext{
*This work was supported by National Natural Science Foundation of China (11771312).

${ }^{\dagger}$ School of Mathematics, Sichuan University, Chengdu 610064, China. Email: wangtao5233@hotmail.com

${ }^{\ddagger}$ School of Science, Chongqing University of Technology, Chongqing 400054, China. Email: yangchaochao9055@163.com

$\S$ Corresponding author. School of Mathematics, Sichuan University, Chengdu 610064, China. Email: xpxie@scu.edu.cn
} 
The extended finite element method (XFEM, also called generalized finite element method(GFEM)) is known to be a widely-used tool for the analysis of problems with singularities [11, 27, 40, 41, 12, 38, 8, 24, 7, 14, 5]. With additional basis functions characterizing the singularity added into the standard approximation space, XFEM does not need body-fitted meshes, and thus avoid complicated meshes.

In this paper, we consider an XFEM for the optimal control (1.1)-(1.3) problem in non-convex domains. We follow the variational discretization concept [21, 22] to discretize the continuous problem. Optimal error estimations are derived for the state, co-state and control.We apply the semi-smooth Newton method to solve the resultant nonlinear discrete system.

The rest of the paper is arranged as follows. Section 2 gives some notations, the optimality conditions, and regularity results for the optimal control problem. Section 3 introduces the XFEM, and shows several theoretical results associate with XFEM. Section 4 is devoted to the discrete optimal control problem, the discrete optimality conditions and error estimates for the state, co-state and control. Section 5 gives an iteration algorithm for the discrete system. Finally, Section 6 provides numerical results to verify the theoretical analysis.

\section{Preliminary}

Let $\Omega$ be a polygonal domain with a single re-entrant corner of angle $\frac{\pi}{\beta}, \beta \in\left[\frac{1}{2}, 1\right]$. And $(0,0)$ is the concave point of $\Omega$. For any nonnegative integer $m$, let $H^{m}(\Omega)$ and $H_{0}^{m}(\Omega)$ denote the standard Sobolev spaces on $\Omega$ with norm $\|\cdot\|_{m}$ and semi-norm $|\cdot|_{m}$. In particular, $H^{0}(\Omega)=L^{2}(\Omega)$, with the standard $L^{2}$-inner product $(\cdot, \cdot)$. that

For $u \in L^{2}(\Omega)$, the weak formulation of the state equation (1.2) is as follows: find $y \in H_{0}^{1}(\Omega)$ such

$$
a(y, v)=(u+f, v), \quad \forall v \in H_{0}^{1}(\Omega)
$$

where $a(y, v):=(\nabla y, \nabla v)$.

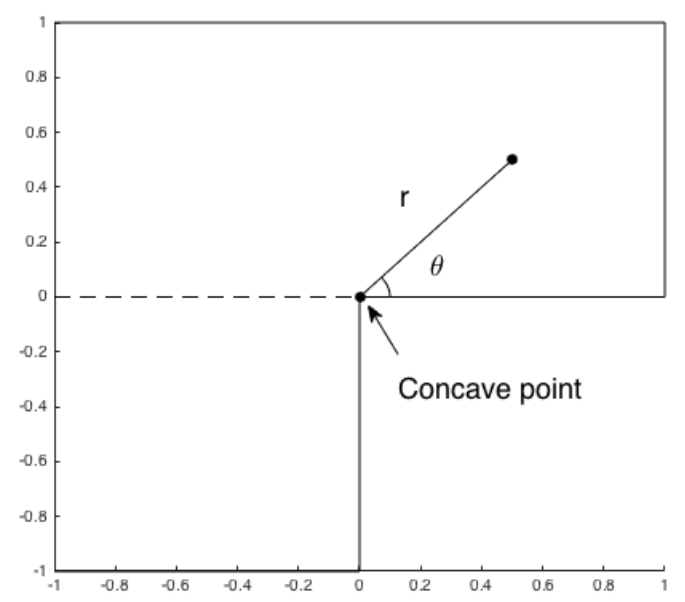

Figure 1: Polar coordinates.

Introduce a singular function

$$
S_{\beta}(r, \theta)=r^{\beta} \sin (\beta \theta) .
$$

It is well known that the weak solution $y$ is generally not in $H^{2}(\Omega)$. According to [15, 19], $y$ has a singular part

$$
y_{s}=\chi(r) K_{y} S_{\beta}(r, \theta)
$$

such that

$$
y-y_{s} \in H^{2}(\Omega)
$$

Here $r$ and $\theta$ are polar coordinates with $0 \leq \theta \leq 2 \pi$ (cf. Figure 1 for an $L$-shaped domain). $K_{y}$ is a positive constant depending on $u$ and can be regarded as a linear functional of $u+f$ (cf. [23, 37]). 
$\chi \in W^{2, \infty}(0, \infty)$ is a cut-off function defined by

$$
\begin{cases}\chi(r)=1, & \text { if } r<r_{0}, \\ 0<\chi(r)<1, & \text { if } r_{0}<r<r_{1}, \\ \chi(r)=0, & \text { if } r_{1}<r,\end{cases}
$$

where $r_{0}, r_{1}$ are two constants with $0<r_{0}<r_{1}$. And, throughout the paper, we use " $p \lesssim q$ " to denote " $p \leq C q$, where $C$ is a generic positive constant independent of the solution $u$ and the finite element mesh size $h$.

In view of (2.2)-(2.3), we assume the following regularity on the solution $y$ to the problem (2.1) (cf. [37]):

$$
K_{y}+\left\|y-y_{s}\right\|_{2} \lesssim\|u\|_{0}+\|f\|_{0}
$$

Remark 2.1. Set

$$
\widetilde{y_{s}}:=K_{y} S_{\beta}(r, \theta) \text {, }
$$

and let $B(r)$ be a ball with center $(0,0)$ and radius $r$. Then, for any given enrichment radius $r_{s}>0$, by (2.5) we easily get

$$
\left\|y-\widetilde{y_{s}}\right\|_{2}+\left\|\widetilde{y_{s}}\right\|_{1}+\left\|\widetilde{y_{s}}\right\|_{2, \Omega \backslash B\left(r_{s} / 2\right)} \lesssim\|u\|_{0}+\|f\|_{0} .
$$

In fact, from (2.5) it follows

$$
\left\|\widetilde{y_{s}}\right\|_{1}+\left\|\widetilde{y_{s}}\right\|_{2, \Omega \backslash B\left(r_{s} / 2\right)}=K_{y}\left(\left\|S_{\beta}\right\|_{1}+\left\|S_{\beta}\right\|_{2, \Omega \backslash B\left(r_{s} / 2\right)}\right) \lesssim\|u\|_{0}+\|f\|_{0} .
$$

By the definition of the cut-off function $\chi(r)$ we have

$$
\left\|(1-\chi) \widetilde{y_{s}}\right\|_{2}=\left\|(1-\chi) \widetilde{y_{s}}\right\|_{2, \Omega \backslash B\left(r_{0}\right)} \leq\left\|\widetilde{y_{s}}\right\|_{2, \Omega \backslash B\left(r_{0}\right)} \lesssim\|u\|_{0}+\|f\|_{0},
$$

which, together with the triangle inequality, yields

$$
\left\|y-\widetilde{y_{s}}\right\|_{2} \leq\left\|y-y_{s}\right\|_{2}+\left\|(1-\chi) \widetilde{y_{s}}\right\|_{2} \lesssim\|u\|_{0}+\|f\|_{0} .
$$

Hence, the estimate (2.7) holds.

By Following the standard optimality technique in [43], we can easily get the optimality conditions of the optimal control problem (1.1)-(1.3).

Lemma 2.1. The optimal control problem (1.1)-(1.3) has a unique solution $(y, p, u) \in H_{0}^{1}(\Omega) \times H_{0}^{1}(\Omega) \times$ $U_{a d}$ such that

$$
\begin{aligned}
& a(y, v)=(u+f, v), \quad \forall v \in H_{0}^{1}(\Omega), \\
& a(v, p)=\left(y-y_{d}, v\right), \quad \forall v \in H_{0}^{1}(\Omega), \\
& (p+a u, v-u) \geq 0, \quad \forall v \in U_{a d}
\end{aligned}
$$

where

$$
U_{a d}:=\left\{v \in L^{2}(\Omega): u_{a} \leq v \leq u_{b} \text { a.e. in } \Omega\right\} .
$$

We note that $p$ is called the co-state or adjoint state, and (2.9) is the co-state equation.

Remark 2.2. Let

$$
p_{s}:=\chi(r) K_{p} S_{\beta}(r, \theta),
$$

with $K_{p}>0$, be the singular part of the solution $p$ to the co-state equation (2.9) such that

$$
K_{p}+\left\|p-p_{s}\right\|_{2} \lesssim\left\|y-y_{d}\right\|_{0}
$$

which, together with the fact $\|y\|_{1} \lesssim\|u\|_{0}$, means

$$
K_{p}+\left\|p-p_{s}\right\|_{2} \lesssim\|u\|_{0}+\left\|y_{d}\right\|_{0}+\|f\|_{0} .
$$

Similar to Remark 2.1, set

$$
\widetilde{p_{s}}:=K_{p} S_{\beta}(r, \theta),
$$

then from (2.14) we have

$$
\left\|p-\widetilde{p_{s}}\right\|_{2}+\left\|\widetilde{p_{s}}\right\|_{1}+\left\|\widetilde{p_{s}}\right\|_{2, \Omega \backslash B\left(r_{s} / 2\right)} \lesssim\|u\|_{0}+\|f\|_{0}+\left\|y_{d}\right\|_{0} .
$$


Remark 2.3. The variational inequality (2.10) means that

$$
u=P_{U_{a d}}\left(-\frac{1}{\alpha} p\right),
$$

where $P_{U_{a d}}$ denotes the $L^{2}$ - projection onto $U_{a d}$.

\section{XFEM for state and co-state equations}

From Lemma 2.1, the state $y$ and the co-state $p$ can respectively be viewed as the solutions to the following two problems.

Find $y \in H_{0}^{1}(\Omega)$ such that

$$
a(y, v)=(u+f, v), \quad \forall v \in H_{0}^{1}(\Omega) .
$$

Find $p \in H_{0}^{1}(\Omega)$ such that

$$
a(v, p)=\left(y-y_{d}, v\right), \quad \forall v \in H_{0}^{1}(\Omega)
$$

\subsection{Formulations of XFEM}

Let $\mathscr{T}_{h}$ be a shape-regular triangulation of $\Omega$ consisting of open triangles with mesh size $h=\max _{K \in \mathscr{T}_{h}} h_{K}$, where $h_{K}$ denotes the diameter of $K \in \mathscr{T}_{h}$. Denote by $\Theta=\left\{a_{i}: i \in 1,2, \cdots, I\right\}$ the set of all the vertexes of all triangles in $\mathscr{T}_{h}$.

For $\forall a_{i} \in \Theta$, let $\varphi_{i}$ be the corresponding nodal basis function of the continuous linear finite element method with respect to $\mathcal{T}_{h}$. Let $r_{s}>0$ be a prescribed constant called enrichment radius. We define a vertex set

$$
\Theta_{S}:=\left\{a_{i} \in \Theta: \text { the distance between } a_{i} \text { and the concave point is less than or equal to } r_{s}\right\} .
$$

In particular, when $\Omega$ is a cracked domain, i.e. $\beta=\frac{1}{2}$, we set

$$
\Theta_{H}:=\left\{a_{i} \in \Theta \text { : the support of } \varphi_{i} \text { is completely cut by the crack of } \Omega\right\},
$$

and define the Heaviside function $H(x)$ : for any $x=(r \cos \theta, r \sin \theta) \in \Omega$,

$$
H(x)= \begin{cases}+1 & \text { if } x \cdot n \geq 0 \\ -1 & \text { if } x \cdot n<0\end{cases}
$$

where $n$ is a unit normal vector along the crack.

With the above notations, set

$$
\begin{gathered}
W_{1}:=\operatorname{span}\left\{\varphi_{i}: a_{i} \in \Theta\right\}+\operatorname{span}\left\{\chi(r) S_{\beta}\right\}, \\
W_{1}^{*}:=\operatorname{span}\left\{\varphi_{i}: a_{i} \in \Theta\right\}+\operatorname{span}\left\{\varphi_{i} H: a_{i} \in \Theta_{H}\right\}+\operatorname{span}\left\{\chi(r) S_{\frac{1}{2}}\right\}, \\
W_{2}:=\operatorname{span}\left\{\varphi_{i}: a_{i} \in \Theta\right\}+\operatorname{span}\left\{\varphi_{i} S_{\beta}: a_{i} \in \Theta_{S}\right\}, \\
W_{2}^{*}:=\operatorname{span}\left\{\varphi_{i}: a_{i} \in \Theta\right\}+\operatorname{span}\left\{\varphi_{i} H: a_{i} \in \Theta_{H}\right\}+\operatorname{span}\left\{\varphi_{i} S_{\beta}: a_{i} \in \Theta_{S}\right\},
\end{gathered}
$$

and $V_{0}:=\{v: v=0$ on $\partial \Omega\}$. Then we introduce the following two extended finite element spaces:

It is easy to observe that

$$
\begin{aligned}
& V_{h}^{1}:= \begin{cases}W_{1} \cap V_{0} & \text { if } \frac{1}{2}<\beta \leq 1, \\
W_{1}^{*} \cap V_{0} & \text { if } \beta=\frac{1}{2},\end{cases} \\
& V_{h}^{2}:= \begin{cases}W_{2} \bigcap V_{0} & \text { if } \frac{1}{2}<\beta \leq 1, \\
W_{2}^{*} \bigcap V_{0} & \text { if } \beta=\frac{1}{2} .\end{cases}
\end{aligned}
$$

$$
V_{h}^{i} \subset H_{0}^{1}(\Omega), \quad i=1,2 .
$$


Take $V_{h}=V_{h}^{1}$ or $V_{h}^{2}$, then the XFEM formulations for the weak problems of the state $y$ and co-state $p$ read as follows:

Find $y^{h} \in V_{h}$ such that

$$
a\left(y^{h}, v_{h}\right)=\left(u+f, v_{h}\right), \quad \forall v_{h} \in V_{h} .
$$

Find $p^{h} \in V_{h}$ such that

$$
a\left(v_{h}, p^{h}\right)=\left(y-y_{d}, v_{h}\right), \quad \forall v_{h} \in V_{h} .
$$

Remark 3.1. The XFEM with $V_{h}=V_{h}^{1}$ was called an XFEM with a cut-off function [28], and the one with $V_{h}=V_{h}^{2}$ was called a classic XFEM with a fixed enrichment area [11].

\subsection{Error estimates of XFEM}

According to [28], it holds the following error estimates.

Lemma 3.1. Let $y, p$ be the solutions to the continuous problems (3.1) and (3.2) respectively such that the regularity conditions (2.5) and (2.14) hold, and $y^{h}, p^{h}$ be the solutions to the discrete schemes (3.4) and (3.5) respectively. Then the estimates

$$
\begin{aligned}
\left\|y-y^{h}\right\|_{1} & \lesssim h\left\|y-y_{s}\right\|_{2} \lesssim h\left(\|u\|_{0}+\|f\|_{0}\right), \\
\left\|p-p^{h}\right\|_{1} & \lesssim h\left\|p-p_{s}\right\|_{2} \lesssim h\left(\|u\|_{0}+\|f\|_{0}+\left\|y_{d}\right\|_{0}\right)
\end{aligned}
$$

hold for $V_{h}=V_{h}^{1}$, and the estimates

$$
\begin{aligned}
\left\|y-y^{h}\right\|_{1} \lesssim h\left(\left\|y-\widetilde{y_{s}}\right\|_{2}+\left\|\widetilde{y_{s}}\right\|_{1}+\left\|\widetilde{y_{s}}\right\|_{2, \Omega \backslash B\left(r_{s} / 2\right)}\right) \lesssim h\left(\|u\|_{0}+\|f\|_{0}\right), \\
\left\|p-p^{h}\right\|_{1} \lesssim h\left(\left\|p-\widetilde{p_{s}}\right\|_{2}+\left\|\widetilde{p_{s}}\right\|_{1}+\left\|\widetilde{p_{s}}\right\|_{2, \Omega \backslash B\left(r_{s} / 2\right)}\right) \lesssim h\left(\|u\|_{0}+\|f\|_{0}+\left\|y_{d}\right\|_{0}\right)
\end{aligned}
$$

hold for $V_{h}=V_{h}^{2}$.

Based on this lemma, we can follow standard duality arguments to derive $L^{2}$ - estimates of the errors $y-y^{h}$ and $p-p^{h}$.

Lemma 3.2. Under the same conditions of Lemma 3.1, the following estimates hold:

$$
\begin{aligned}
& \left\|y-y^{h}\right\|_{0} \lesssim h^{2}\left(\|u\|_{0}+\|f\|_{0}\right), \\
& \left\|p-p^{h}\right\|_{0} \lesssim h^{2}\left(\|u\|_{0}+\|f\|_{0}+\left\|y_{d}\right\|_{0}\right) .
\end{aligned}
$$

Proof. We only show (3.10) for $V_{h}=V_{h}^{1}$, since the other cases follow similarly.

Consider the auxiliary problem

$$
\left\{\begin{aligned}
-\Delta z & =y-y^{h} & & \text { in } \Omega \\
z & =0 & & \text { on } \partial \Omega
\end{aligned}\right.
$$

which indicates

$$
a(z, v)=\left(y-y^{h}, v\right), \quad \forall v \in H_{0}^{1}(\Omega) .
$$

Let $z^{h} \in V_{h}^{1}$ satisfy

$$
a\left(z^{h}, v_{h}\right)=\left(y-y^{h}, v_{h}\right), \quad \forall v_{h} \in V_{h}^{1},
$$

and $z_{s}:=\chi(r) K_{z} S_{\beta}(r, \theta)$ be the regular part of $z$ with

$$
K_{z}+\left\|z-z_{s}\right\|_{2} \lesssim\left\|y-y^{h}\right\|_{0}
$$

Then, similar to (3.6), it holds

$$
\left\|z-z^{h}\right\|_{1} \lesssim h\left\|z-z_{s}\right\|_{2} \lesssim h\left\|y-y^{h}\right\|_{0} .
$$

As a result, by the Galerkin orthogonality $a\left(z^{h}, y-y^{h}\right)=0$ and (3.6) we have

$$
\begin{aligned}
\left\|y-y^{h}\right\|_{0}^{2} & =a\left(z-z^{h}, y-y^{h}\right) \\
& \leq\left\|z-z^{h}\right\|_{1}\left\|y-y^{h}\right\|_{1} \\
& \lesssim h^{2}\left\|y-y^{h}\right\|_{0}\left(\|u\|_{0}+\|f\|_{0}\right),
\end{aligned}
$$

which yields (3.10). 


\section{Discrete optimal control problem}

\subsection{Discrete optimality conditions}

In this subsection, we follow the variational discretization concept [21] to discretize the optimal control problem (1.1)-(1.3). The corresponding discrete optimal control problem is of the form

$$
\min _{\left(y_{h}, u\right) \in V_{h} \times U_{a d}} J_{h}\left(y_{h}, u\right)=\frac{1}{2} \int_{\Omega}\left(y_{h}-y_{d}\right)^{2} d s+\frac{\alpha}{2} \int_{\Omega} u^{2} d s
$$

where $y_{h}=y_{h}(u)$ satisfies

$$
a\left(y_{h}, v_{h}\right)=\left(u+f, v_{h}\right), \quad \forall v_{h} \in V_{h} .
$$

Similar to the continuous case, we have the following existence and uniqueness result and optimality conditions.

Lemma 4.1. The discrete optimal control problem (4.1)-(4.2) admits a unique solution $\left(y_{h}, u_{h}\right) \in V_{h} \times$ $U_{a d}$, and its equivalent optimality conditions read: find $\left(y_{h}, p_{h}, u_{h}\right) \in V_{h} \times V_{h} \times U_{a d}$ such that

$$
\begin{aligned}
& a\left(y_{h}, v_{h}\right)=\left(u_{h}+f, v_{h}\right), \quad \forall v_{h} \in V_{h}, \\
& a\left(v_{h}, p_{h}\right)=\left(y_{h}-y_{d}, v_{h}\right), \quad \forall v_{h} \in V_{h}, \\
& \left(p_{h}+\alpha u_{h}, v-u_{h}\right) \geq 0, \quad \forall v \in U_{a d} .
\end{aligned}
$$

Remark 4.1. We note that the optimal control $u$ is not directly discretized in the objective functional (4.1), as $U_{a d}$ is infinite dimensional. In fact, the variational inequality (4.5) means that the discrete control $u_{h}$ is the $L^{2}-$ projection of $-\frac{p_{h}}{\alpha}$ onto $U_{a d}$, i.e.

$$
u_{h}=P_{U_{a d}}\left(-\frac{p_{h}}{\alpha}\right)
$$

This is a key point of the variational discretization concept. In particular, if the functions $u_{0}$ and $u_{1}$ are well-defined at any $x \in \Omega$, then (4.6) is equivalent to

$$
u_{h}=\min \left\{u_{1}, \max \left\{u_{0},-\frac{p_{h}}{\alpha}\right\}\right\} .
$$

\subsection{Error estimates}

Recall that $y^{h} \in V_{h}$ and $p^{h} \in V_{h}$ are the solutions to the XFEM formulations (3.4) and (3.5), respectively. In what follows we first show that the errors between $(y, p, u)$ and $\left(y_{h}, p_{h}, u_{h}\right)$, which are the solutions of the continuous optimal control problem (2.8)-(2.10) and the discrete optimal control problem (4.2)-(4.5) respectively, are bounded from above by the errors between $(y, p)$ and $\left(y^{h}, p^{h}\right)$.

Theorem 4.1. Let $(y, p, u) \in H_{0}^{1}(\Omega) \times H_{0}^{1}(\Omega) \times U_{a d}$ and $\left(y_{h}, p_{h}, u_{h}\right) \in V_{h} \times V_{h} \times U_{a d}$ be the solutions to the continuous problem (2.8)-(2.10) and the discrete problem (4.2)-(4.5), respectively. Then we have

$$
\begin{aligned}
\alpha^{\frac{1}{2}}\left\|u-u_{h}\right\|_{0}+\left\|y-y_{h}\right\|_{0} & \lesssim\left\|y-y^{h}\right\|_{0}+\alpha^{-\frac{1}{2}}\left\|p-p^{h}\right\|_{0}, \\
\left\|p-p_{h}\right\|_{0} & \lesssim\left\|p-p^{h}\right\|_{0}+\left\|y-y_{h}\right\|_{0}, \\
\left|y-y_{h}\right|_{1} & \lesssim\left|y-y^{h}\right|_{1}+\left\|u-u_{h}\right\|_{0}, \\
\left|p-p_{h}\right|_{1} & \lesssim\left|p-p^{h}\right|_{1}+\left\|y-y_{h}\right\|_{0} .
\end{aligned}
$$

Proof. We first show (4.8). From (3.4)-(3.5) and (4.2)-(4.4) it follows

$$
\begin{aligned}
& a\left(y_{h}-y^{h}, v_{h}\right)=\left(u_{h}-u, v_{h}\right), \quad \forall v_{h} \in V_{h}, \\
& a\left(v_{h}, p_{h}-p^{h}\right)=\left(y_{h}-y, v_{h}\right), \quad \forall v_{h} \in V_{h},
\end{aligned}
$$

which yields

$$
\left(y_{h}-y, y_{h}-y^{h}\right)=a\left(y_{h}-y^{h}, p_{h}-p^{h}\right)=\left(u_{h}-u, p_{h}-p^{h}\right)
$$


By (2.10) and (4.5) we get

$$
\left(\alpha u+p, u_{h}-u\right) \geq 0, \quad\left(\alpha u_{h}+p_{h}, u-u_{h}\right) \geq 0,
$$

which imply

$$
\left(\alpha\left(u-u_{h}\right)+p-p_{h}, u_{h}-u\right) \geq 0 .
$$

This inequality, together with (4.14), indicates

$$
\begin{aligned}
\alpha\left\|u-u_{h}\right\|_{0}^{2} & \leq\left(u_{h}-u, p-p_{h}\right) \\
& =\left(u_{h}-u, p-p^{h}\right)+\left(u_{h}-u, p^{h}-p_{h}\right) \\
& =\left(u_{h}-u, p-p^{h}\right)+\left(y_{h}-y, y^{h}-y_{h}\right) \\
& \leq \frac{1}{2}\left(\alpha\left\|u-u_{h}\right\|_{0}^{2}+\frac{1}{\alpha}\left\|p-p^{h}\right\|_{0}^{2}\right)-\left(y-y_{h}, y-y_{h}\right)+\left(y_{h}-y, y^{h}-y\right) \\
& \leq \frac{1}{2}\left(\alpha\left\|u-u_{h}\right\|_{0}^{2}+\frac{1}{\alpha}\left\|p-p^{h}\right\|_{0}^{2}\right)-\frac{1}{2}\left\|y-y_{h}\right\|_{0}^{2}+\frac{1}{2}\left\|y-y^{h}\right\|_{0}^{2},
\end{aligned}
$$

which implies (4.8).

Secondly, let us prove (4.9). Since $p_{h}-p^{h} \in V_{h} \subset H_{0}^{1}(\Omega)$ (cf. (3.3)), by (4.13) we have

$$
\begin{aligned}
\left\|p_{h}-p^{h}\right\|_{0}^{2} & \lesssim\left|p_{h}-p^{h}\right|_{1}^{2} \\
& =a\left(p_{h}-p^{h}, p_{h}-p^{h}\right)=\left(y_{h}-y, p_{h}-p^{h}\right) \\
& \lesssim\left\|y_{h}-y\right\|_{0}\left\|p_{h}-p^{h}\right\|_{0},
\end{aligned}
$$

which, together with the triangle inequality, leads to

$$
\begin{aligned}
\left\|p-p_{h}\right\|_{0} & \leq\left\|p-p^{h}\right\|_{0}+\left\|p^{h}-p_{h}\right\|_{0} \\
& \lesssim\left\|p-p^{h}\right\|_{0}+\left\|y_{h}-y\right\|_{0},
\end{aligned}
$$

i.e. (4.9) holds.

Thirdly, let us derive (4.10). In view of (4.12), we obtain

$$
\begin{aligned}
\left|y_{h}-y^{h}\right|_{1}^{2} & =a\left(y_{h}-y^{h}, y_{h}-y^{h}\right)=\left(u_{h}-u, y_{h}-y^{h}\right) \\
& \leq\left\|u-u_{h}\right\|_{0}\left\|y_{h}-y^{h}\right\|_{0} \\
& \lesssim\left\|u-u_{h}\right\|_{0}\left|y_{h}-y^{h}\right|_{1},
\end{aligned}
$$

which, together with the triangle inequality, indicates (4.10).

Finally, let us show (4.11).From (4.13) we get

$$
\begin{aligned}
\left|p_{h}-p^{h}\right|_{1}^{2} & =a\left(p_{h}-p^{h}, p_{h}-p^{h}\right)=\left(y_{h}-y, p_{h}-p^{h}\right) \\
& \lesssim\left\|y_{h}-y\right\|_{0}|| p_{h}-p^{h} \|_{0} \\
& \lesssim\left\|y_{h}-y\right\|_{0}\left|p_{h}-p^{h}\right|_{1},
\end{aligned}
$$

which, together with the triangle inequality, yields (4.11).

Based on Theorem 4.1 and Lemmas 3.1-3.2, we immediately have the following optimal error estimates.

Theorem 4.2. Let $(y, p, u) \in H_{0}^{1}(\Omega) \times H_{0}^{1}(\Omega) \times U_{a d}$ and $\left(y_{h}, p_{h}, u_{h}\right) \in V_{h} \times V_{h} \times U_{a d}$ be the solutions to the continuous problem (2.8)-(2.10) and the discrete problem (4.2)-(4.5) respectively such that the regularity conditions (2.5) and (2.14) hold. Then we have

$$
\begin{aligned}
\left\|u-u_{h}\right\|_{0}+\left\|y-y_{h}\right\|_{0}+\left\|p-p_{h}\right\|_{0} & \lesssim h^{2}\left(\|u\|_{0}+\|f\|_{0}+\left\|y_{d}\right\|_{0}\right), \\
\left|y-y_{h}\right|_{1}+\left|p-p_{h}\right|_{1} & \lesssim h\left(\|u\|_{0}+\|f\|_{0}+\left\|y_{d}\right\|_{0}\right) .
\end{aligned}
$$




\section{Iteration algorithm}

Notice that the optimal control problem (1.1)-(1.2) without the constraint (1.3) is a linear problem, and the resultant discrete linear system is easy to solve. However, for the constrained optimal control problem (1.1)-(1.3), the corresponding discrete optimal control problem (4.1)-(4.2) or its equivalent optimality problem (4.3)-(4.5) is a nonlinear system, and we shall apply the semi-smooth Newton algorithm [22] to solve it. To describe this iteration algorithm, we first show the matrix form of the discrete system (4.3)-(4.5).

Let $\left\{\varphi_{i}: i=1,2, \cdots, I\right\}$ be a set of basis functions of the XFE space $V_{h}$ with $I=\operatorname{dim}\left(V_{h}\right)$, and $Y_{h}, P_{h}$ be column vectors consisting of corresponding degrees of freedom of $y_{h}, p_{h}$ respectively, such that

$$
y_{h}=\left(\varphi_{1}, \varphi_{2}, \cdots, \varphi_{I}\right) Y_{h}, \quad p_{h}=\left(\varphi_{1}, \varphi_{2}, \cdots, \varphi_{I}\right) P_{h}
$$

Define matrices $A, M \in \Re^{I \times I}$ and vectors $F_{1}, F_{2} \in \Re^{I}$ by

$$
\begin{aligned}
& A(i, j)=a\left(\varphi_{i}, \varphi_{j}\right), \quad M(i, j)=\left(\varphi_{i}, \varphi_{j}\right), \\
& F_{1}(j)=\left(f, \varphi_{j}\right), \quad F_{2}(j)=\left(y_{d}, \varphi_{j}\right)
\end{aligned}
$$

for $i, j=1,2, \cdots, I$. Then (4.3) and (4.4) are equivalent to the following matrix equations:

$$
\begin{aligned}
& A Y_{h}=M U_{h}+F_{1}, \\
& A P_{h}=M Y_{h}-F_{2} .
\end{aligned}
$$

In view of $(4.7)$, i.e. $u_{h}=\min \left\{u_{1}, \max \left\{u_{0},-\frac{p_{h}}{\alpha}\right\}\right\}$, we define the in-active set $\Omega_{I}$ and active set $\Omega_{A}$ as follows:

$$
\begin{gathered}
\Omega_{I}:=\left\{x \in \Omega: u_{0}<-\frac{p_{h}}{\alpha}<u_{1}\right\}, \\
\Omega_{A}=\Omega_{A 0} \cup \Omega_{A 1}, \quad \Omega_{A 0}:=\left\{x \in \Omega:-\frac{p_{h}}{\alpha} \leq u_{0}\right\}, \Omega_{A 1}:=\left\{x \in \Omega:-\frac{p_{h}}{\alpha} \geq u_{1}\right\} .
\end{gathered}
$$

It is evident that

$$
\left.u_{h}\right|_{\Omega_{I}}=-\frac{p_{h}}{\alpha},\left.\quad u_{h}\right|_{\Omega_{A 0}}=u_{0},\left.\quad u_{h}\right|_{\Omega_{A 1}}=u_{1} .
$$

Note that in the algorithm to be given $u_{0}, u_{1}$ will be replaced by their approximations.

Let $V_{h}^{*}$ be the modified finite element space of $V_{h}$,

$$
\begin{aligned}
& V_{h}^{1, *}:= \begin{cases}W_{1} & \text { if } \frac{1}{2}<\beta \leq 1, \\
W_{1}^{*} & \text { if } \beta=\frac{1}{2},\end{cases} \\
& V_{h}^{2, *}:= \begin{cases}W_{2} & \text { if } \frac{1}{2}<\beta \leq 1, \\
W_{2}^{*} & \text { if } \beta=\frac{1}{2} .\end{cases}
\end{aligned}
$$

$u_{0}^{*}, u_{1}^{*} \in V_{h}^{*}$ are $L^{2}-$ projections of $u_{0}, u_{1}$ onto $V_{h}^{*}$, respectively. Let $u_{h}^{*}$ be an approximation of $u_{h}$ with

$$
\left.u_{h}^{*}\right|_{\Omega_{I}}=u_{h},\left.\quad u_{h}^{*}\right|_{\Omega_{A 0}}=u_{0}^{*},\left.\quad u_{h}^{*}\right|_{\Omega_{A 1}}=u_{1}^{*} .
$$

It is obvious that $u_{h}^{*} \in \phi V_{h}^{*}+(1-\phi) V_{h}^{*}$, where $\phi$ is the characteristic function of $\Omega_{I}$. Let $U_{h, 1}, U_{h, 2} \in$ $\Re^{I}$ denote the column vectors consisting of corresponding degrees of freedom of $\phi u_{h}^{*}$ and $(1-\phi) u_{h}^{*}$, respectively, and define matrices $M_{1}, M_{2} \in \Re^{I \times I}$ by

$$
M_{1}(i, j):=\left(\varphi_{i}, \phi \varphi_{j}\right), \quad M_{2}(i, j):=\left(\varphi_{i},(1-\phi) \varphi_{j}\right)
$$

for $i=1,2, \cdots, I$ and $j=1,2, \cdots, I$. Then the matrix form (5.1) is modified as

$$
A Y_{h}=M_{1} U_{h, 1}+M_{2} U_{h, 2}+F_{1}
$$

Based on (5.1)-(5.5), we can describe the semi-smooth newton algorithm as follows. 


\section{Semi-smooth newton algorithm}

Set $k=0, \phi^{(0)}=0, U_{h, 1}^{(0)}=0, U_{h, 2}^{(0)}=0$;

\section{Do until convergence}

1. Compute $y_{h}^{(k+1)} \in V^{h}$ by

$$
a\left(y_{h}^{(k+1)}, v_{h}\right)=\left(\phi^{(k)} u_{h}^{(k)}+\left(1-\phi^{(k)}\right) u_{h}^{(k)}+f, v_{h}\right), \forall v_{h} \in V^{h}
$$

or, equivalently, compute

$$
Y_{h}^{(k+1)}=A^{-1}\left(M_{1}^{(k)} U_{h, 1}^{(k)}+M_{2}^{(k)} U_{h, 2}^{(k)}+F_{1}\right)
$$

2. Compute $p_{h}^{(k+1)} \in V^{h}$ by

$$
a\left(v_{h}, p_{h}^{(k+1)}\right)=\left(y_{h}^{(k+1)}-y_{d}, v_{h}\right), \forall v_{h} \in V^{h}
$$

or, equivalently, compute

$$
P_{h}^{(k+1)}=A^{-1}\left(M Y^{(k+1)}-F_{2}\right)=A^{-1}\left(M A^{-1}\left(M_{1}^{(k)} U_{h, 1}^{(k)}+M_{2}^{(k)} U_{h, 2}^{(k)}+F_{1}\right)-F_{2}\right) ;
$$

3. Compute

$$
\begin{gathered}
\Omega_{I}^{(k+1)}:=\left\{x \in \Omega: u_{0}<-\frac{p_{h}^{(k+1)}}{\alpha}<u_{1}\right\}, \\
\Omega_{A 0}^{(k+1)}:=\left\{x \in \Omega:-\frac{p_{h}^{(k+1)}}{\alpha} \leq u_{0}\right\}, \quad \Omega_{A 1}^{(k+1)}:=\left\{x \in \Omega:-\frac{p_{h}^{(k+1)}}{\alpha} \geq u_{1}\right\},
\end{gathered}
$$

and the characteristic function, $\phi^{(k+1)}$, of $\Omega_{I}^{(k+1)}$;

4. Compute $u_{h, 2}^{(k+1)}$ (or $U_{h, 2}^{(k+1)}$ ) with

$$
\left.u_{h, 2}^{(k+1)}\right|_{\Omega_{A 0}^{(k+1)}}=u_{0}^{*},\left.\quad u_{h, 2}^{(k+1)}\right|_{\Omega_{A 1}^{(k+1)}}=u_{1}^{*},\left.\quad u_{h, 2}^{(k+1)}\right|_{\Omega_{I}^{(k+1)}}=0
$$

5. Compute $U_{h, 1}^{k+1}$ by

$$
U_{h, 1}^{k+1}=-\frac{1}{\alpha}\left(A^{-1}\left(M A^{-1}\left(M_{1}^{(k+1)} U_{h, 1}^{(k+1)}+M_{2}^{(k+1)} U_{h, 2}^{(k+1)}+F_{1}\right)-F_{2}\right)\right),
$$

i.e. $U_{h, 1}^{k+1}=-\frac{1}{\alpha}\left(I+\frac{1}{\alpha} A^{-1} M A^{-1} M_{1}^{(k+1)}\right)^{-1}\left(A^{-1} M A^{-1} M_{2} U_{h, 2}^{(k+1)}+A^{-1} M A^{-1} F_{1}-A^{-1} F_{2}\right)$;

6. $k=k+1$;

end

It should be pointed out that in step 3, the active set can only be computed approximately for XFEM, even when $u_{0}, u_{1}$ are constants, since some basis functions of the XFE spaces are non-linear. In actual computation we just use their piecewise linear interpolations to replace the nonlinear basis functions so as to compute the approximate active set. We refer to [36] for an efficient method to compute the active set for high order finite element methods.

\section{Numerical results}

In this section, we shall provide several numerical examples to verify the performance of the proposed methods, i.e. the discrete schemes (4.1)-(4.2) or (4.3)-(4.5) with $V_{h}=V_{h}^{1}$ and $V_{h}=V_{h}^{2}$. We recall, cf. Remark 3.1, that $V_{h}^{1}$ and $V_{h}^{2}$ are corresponding to the XFEM with a cut-off function (abbr. cut XFEM) and the classic XFEM with a fixed enrichment area (abbr. classic XFEM). 
Example 6.1. An unconstrained problem in a crack domain.

Take $\Omega=[-1,1] \times[-1,1]$ with a segment crack from the point $(-1,0)$ to the crack-tip $(0,0)(\mathrm{cf}$. Figure 2). We choose $\alpha=0.01$, the enrichment radius $r_{s}=0.5$ (cf. Remark 2.1), and the cut-off function $\chi(r)$ in $(2.4)$ is a polynomial with $r_{0}=0.01$ and $r_{1}=0.99$. Let

$$
\begin{aligned}
& y=\sqrt{r} \sin \left(\frac{\theta}{2}\right)-\frac{1}{4} r^{2} \\
& p=x_{2}^{2}\left(1-x_{2}^{2}\right)\left(1-x_{1}^{2}\right)+\frac{1}{2} \sqrt{r} \sin \left(\frac{\theta}{2}\right)\left(1-x_{1}^{2}\right)\left(1-x_{2}^{2}\right), \\
& u=-\frac{p}{\alpha}
\end{aligned}
$$

be the analytical state, co-state and control of the optimal control problem (1.1) subject to

$$
\begin{cases}-\Delta y=u+f, & \text { in } \Omega \\ y=y_{b}, & \text { on } \partial \Omega .\end{cases}
$$

Note that in this case $U_{a d}=L^{2}(\Omega)$, and $y_{d}$ can be obtained by $-\Delta p=y-y_{d}$. In particular, the discrete equation (4.5) yields $u_{h}=-\frac{p_{h}}{\alpha}$, which means $u-u_{h}=-\frac{p-p_{h}}{\alpha}$.

We use $N \times N$ uniform triangular meshes (cf. Figure 2). Tables 1-2 show results of the relative errors between $\left(y_{h}, p_{h}\right)$ and $(y, p)$ in $H^{1}$ semi-norm and $L^{2}$ norm, and Figure 6 shows the relative errors against the mesh size $h=2 / N$. We can see that the proposed methods yield optimal convergence orders, i.e. first order rates of convergence for $\left|y-y_{h}\right|_{1}$ and $\left|p-p_{h}\right|_{1}$, and second order rates of convergence for $\left|y-y_{h}\right|_{0}$ and $\left|p-p_{h}\right|_{0}$. This is consistent with our theoretical results in Theorem 4.2.

For comparison we also show in Table 3 and Figure 4 the relative errors of $\left(y_{h}, p_{h}\right)$ for the standard linear element method $\left(P_{1}\right.$ FEM) on body fitted meshes (cf. Figure 2). We can see that $P_{1}$ FEM yields only about 0.5 order convergence rates for $\left|y-y_{h}\right|_{1}$ and first order convergence rates for $\left|y-y_{h}\right|_{0}$ and $\left|p-p_{h}\right|_{0}$. This is conformable to the theoretical results in [9].
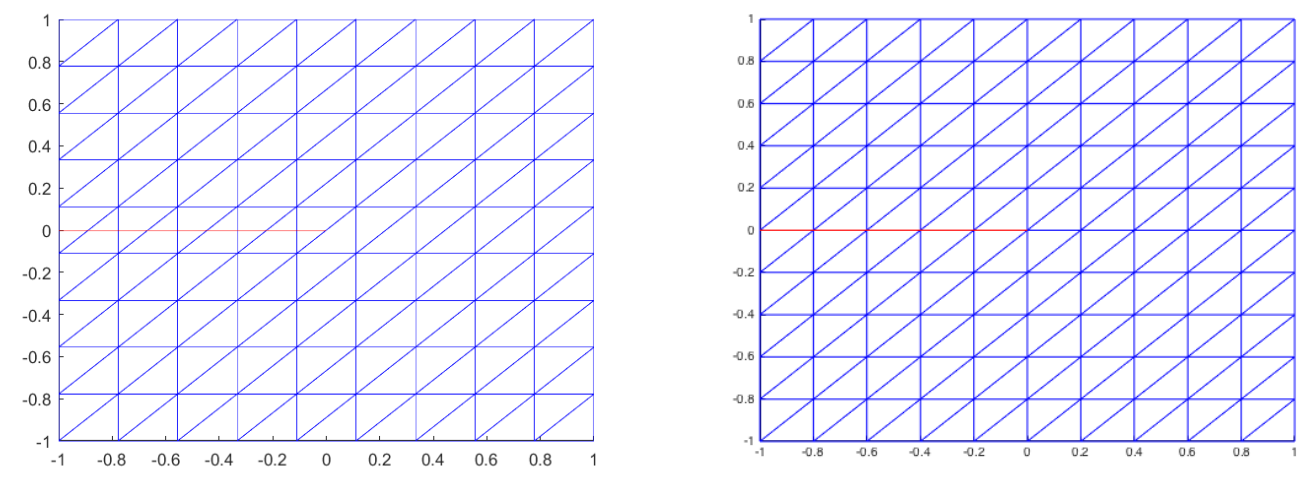

Figure 2: Domain $\Omega$ ( (the red line is the crack) and meshes for Example 6.1: $9 \times 9$ mesh for XFEMs (left) and $10 \times 10$ mesh for $P_{1}$ FEM.

Table 1: Relative errors of the cut XFEM for Example 6.1.

\begin{tabular}{|c|c|c|c|c|c|c|c|c|}
\hline $\mathrm{N}$ & $\frac{\left|y-y_{h}\right|_{1}}{|y|_{1}}$ & order & $\frac{\left\|y-y_{h}\right\|_{0}}{\|y\|_{0}}$ & order & $\frac{\left|p-p_{h}\right|_{1}}{|p|_{1}}$ & order & $\frac{\left\|p-p_{h}\right\|_{0}}{\|p\|_{0}}$ & order \\
\hline 39 & 0.1153 & & 0.0235 & & 0.1457 & & 0.0092 & \\
\hline 49 & 0.0912 & 1.03 & 0.0148 & 2.02 & 0.1162 & 0.99 & 0.0058 & 1.99 \\
\hline 59 & 0.0756 & 1.01 & 0.0101 & 2.06 & 0.0966 & 0.99 & 0.0040 & 2.00 \\
\hline 69 & 0.0647 & 0.99 & 0.0073 & 2.10 & 0.0827 & 0.99 & 0.0029 & 2.01 \\
\hline 79 & 0.0566 & 0.99 & 0.0054 & 2.14 & 0.0723 & 0.99 & 0.0022 & 2.02 \\
\hline
\end{tabular}

Example 6.2. A constrained problem in a crack domain. 
Table 2: Relative errors of the classic XFEM for Example 6.1.

\begin{tabular}{|c|c|c|c|c|c|c|c|c|}
\hline $\mathrm{N}$ & $\frac{\left|y-y_{h}\right|_{1}}{|y|_{1}}$ & order & $\frac{\left\|y-y_{h}\right\|_{0}}{\|y\|_{0}}$ & order & $\frac{\left|p-p_{h}\right|_{1}}{|p|_{1}}$ & order & $\frac{\left\|p-p_{h}\right\|_{0}}{\|p\|_{0}}$ & order \\
\hline 39 & 0.0632 & & 0.0192 & & 0.1380 & & 0.0084 & \\
\hline 49 & 0.0491 & 1.11 & 0.0124 & 1.91 & 0.1103 & 0.98 & 0.0053 & 1.98 \\
\hline 59 & 0.0403 & 1.06 & 0.0087 & 1.93 & 0.0919 & 0.99 & 0.0037 & 1.98 \\
\hline 69 & 0.0344 & 1.02 & 0.0064 & 1.94 & 0.0787 & 0.99 & 0.0027 & 1.99 \\
\hline 79 & 0.0288 & 1.29 & 0.0048 & 2.11 & 0.0685 & 1.02 & 0.0021 & 2.02 \\
\hline
\end{tabular}
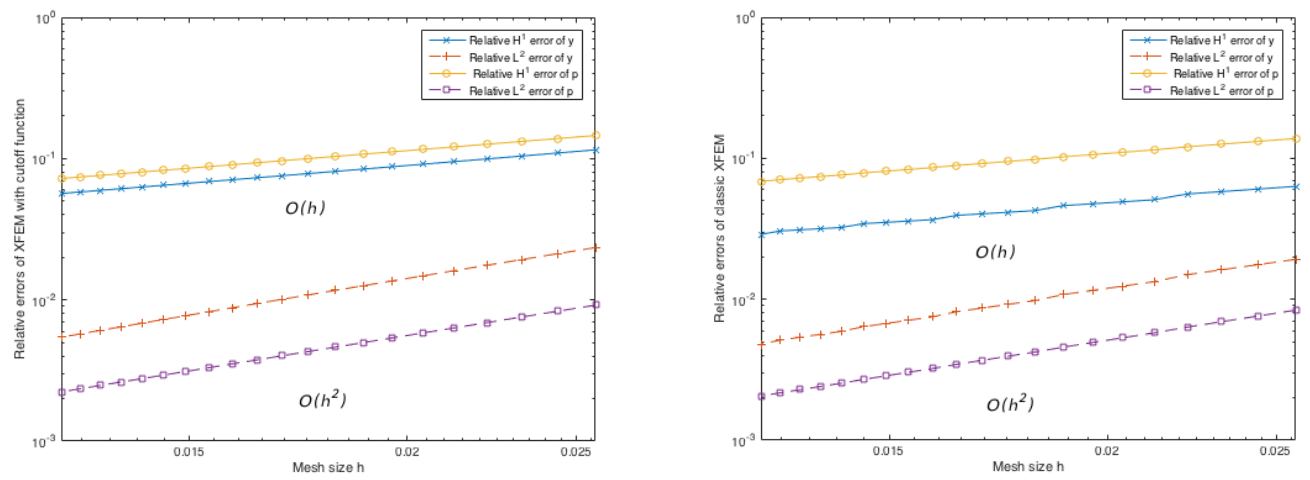

Figure 3: Convergence history of the cut XFEM (left) and classic XFEM (right) for Example 6.1.

Table 3: Relative errors of $P_{1}$ FEM for Example 6.1.

\begin{tabular}{|c|c|c|c|c|c|c|c|c|}
\hline $\mathrm{N}$ & $\frac{\left|y-y_{h}\right|_{1}}{|y|_{1}}$ & order & $\frac{\left\|y-y_{h}\right\|_{0}}{\|y\|_{0}}$ & order & $\frac{\left|p-p_{h}\right|_{1}}{|p|_{1}}$ & order & $\frac{\left\|p-p_{h}\right\|_{0}}{\|p\|_{0}}$ & order \\
\hline 40 & 0.2787 & & 0.0811 & & 0.1779 & & 0.0206 & \\
\hline 50 & 0.2432 & 0.61 & 0.0627 & 1.16 & 0.1492 & 0.79 & 0.0159 & 1.16 \\
\hline 60 & 0.2186 & 0.59 & 0.0511 & 1.13 & 0.1299 & 0.76 & 0.0130 & 1.11 \\
\hline 70 & 0.2002 & 0.57 & 0.0431 & 1.11 & 0.1158 & 0.74 & 0.0110 & 1.09 \\
\hline 80 & 0.1857 & 0.56 & 0.0372 & 1.09 & 0.1051 & 0.72 & 0.0095 & 1.07 \\
\hline
\end{tabular}
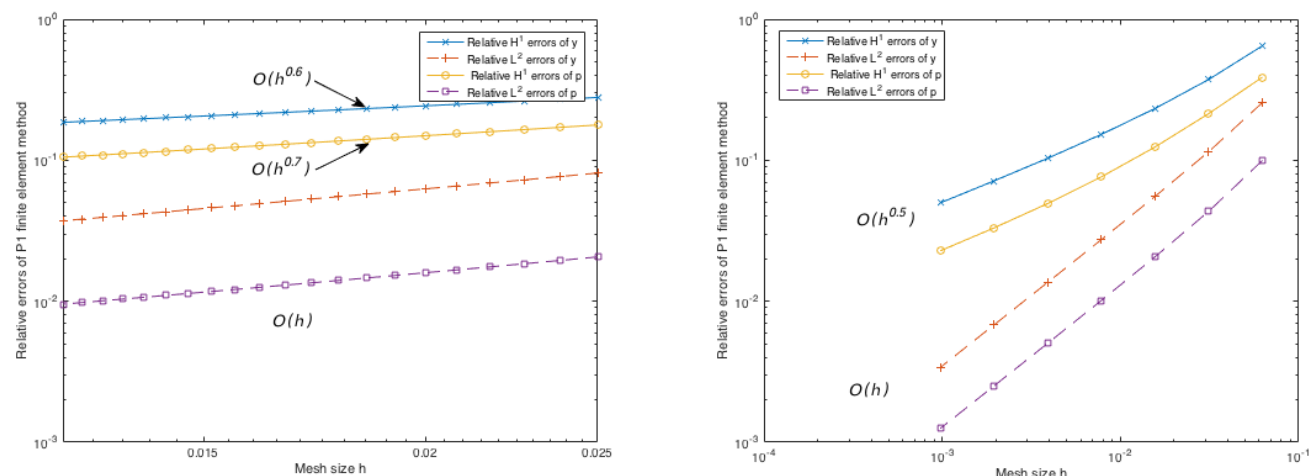

Figure 4: Convergence history of $P_{1}$ FEM for Example 6.1. 
Let the domain $\Omega$, the enrichment radius $r_{s}$, and the cut-off function $\chi(r)$ be the same as in Example 6.1. We take $\alpha=1, u_{0}=-\frac{1}{5}, u_{1}=\frac{1}{5}$, and let

$$
\begin{aligned}
& y=\sqrt{r} \sin \left(\frac{\theta}{2}\right)-\frac{1}{4} r^{2} \\
& p=x_{2}^{2}\left(1-x_{2}^{2}\right)\left(1-x_{1}^{2}\right)+\frac{1}{2} \sqrt{r} \sin \left(\frac{\theta}{2}\right)\left(1-x_{1}^{2}\right)\left(1-x_{2}^{2}\right) \\
& u=\min \left\{\frac{1}{5}, \max \left\{-\frac{p}{\alpha},-\frac{1}{5}\right\}\right\}
\end{aligned}
$$

be the analytical state, co-state and control of the optimal control problem (1.1) subject to (6.1). We use the same meshes as in Example 6.1.

Tables 4-5 show results of the relative errors between $\left(y_{h}, p_{h}, u_{h}\right)$ and $(y, p, u)$, and Figure 5 shows the relative errors against the mesh size $h=2 / N$. We can see that the proposed methods yield optimal convergence orders, i.e. first order rates of convergence for $\left|y-y_{h}\right|_{1}$ and $\left|p-p_{h}\right|_{1}$, and second order rates of convergence for $\left|y-y_{h}\right|_{0},\left|p-p_{h}\right|_{0}$, and $\left|u-u_{h}\right|_{0}$. This is consistent with the theoretical results in Theorem 4.2.

Table 4: Relative errors of the cut XFEM for Example 6.2.

\begin{tabular}{|c|c|c|c|c|c|c|c|c|c|c|}
\hline $\mathrm{N}$ & $\frac{\left|y-y_{h}\right|_{1}}{|y|_{1}}$ & order & $\frac{\left\|y-y_{h}\right\|_{0}}{\|y\|_{0}}$ & order & $\frac{\left|p-p_{h}\right|_{1}}{|p|_{1}}$ & order & $\frac{\left\|p-p_{h}\right\|_{0}}{\|p\|_{0}}$ & order & $\frac{\left\|u-u_{h}\right\|_{0}}{\|u\|_{0}}$ & order \\
\hline 39 & 0.1089 & & 0.0067 & & 0.1456 & & 0.0106 & & 0.0108 & \\
\hline 49 & 0.0880 & 0.94 & 0.0044 & 1.85 & 0.1162 & 0.99 & 0.0067 & 2.00 & 0.0068 & 2.02 \\
\hline 59 & 0.0738 & 0.94 & 0.0031 & 1.89 & 0.0966 & 0.99 & 0.0046 & 2.02 & 0.0047 & 1.97 \\
\hline 69 & 0.0636 & 0.95 & 0.0023 & 1.89 & 0.0827 & 0.99 & 0.0034 & 2.04 & 0.0034 & 2.05 \\
\hline 79 & 0.0559 & 0.96 & 0.0018 & 1.92 & 0.0723 & 0.99 & 0.0026 & 2.06 & 0.0026 & 2.00 \\
\hline
\end{tabular}

Table 5: Relative errors of the classic XFEM for Example 6.2.

\begin{tabular}{|c|c|c|c|c|c|c|c|c|c|c|}
\hline $\mathrm{N}$ & $\frac{\left|y-y_{h}\right|_{1}}{|y|_{1}}$ & order & $\frac{\left\|y-y_{h}\right\|_{0}}{\|y\|_{0}}$ & order & $\frac{\left|p-p_{h}\right|_{1}}{|p|_{1}}$ & order & $\frac{\left\|p-p_{h}\right\|_{0}}{\|p\|_{0}}$ & order & $\frac{\left\|u-u_{h}\right\|_{0}}{\|u\|_{0}}$ & order \\
\hline 39 & 0.0545 & & 0.0024 & & 0.1380 & & 0.0093 & & 0.0099 & \\
\hline 49 & 0.0445 & 0.88 & 0.0015 & 1.90 & 0.1103 & 0.98 & 0.0059 & 1.97 & 0.0062 & 2.02 \\
\hline 59 & 0.0377 & 0.90 & 0.0011 & 1.91 & 0.0919 & 0.99 & 0.0041 & 1.97 & 0.0043 & 1.96 \\
\hline 69 & 0.0327 & 0.91 & 0.0008 & 1.91 & 0.0787 & 0.99 & 0.0030 & 1.98 & 0.0031 & 2.02 \\
\hline 79 & 0.0277 & 1.22 & 0.0006 & 2.23 & 0.0685 & 1.02 & 0.0023 & 2.04 & 0.0024 & 1.99 \\
\hline
\end{tabular}
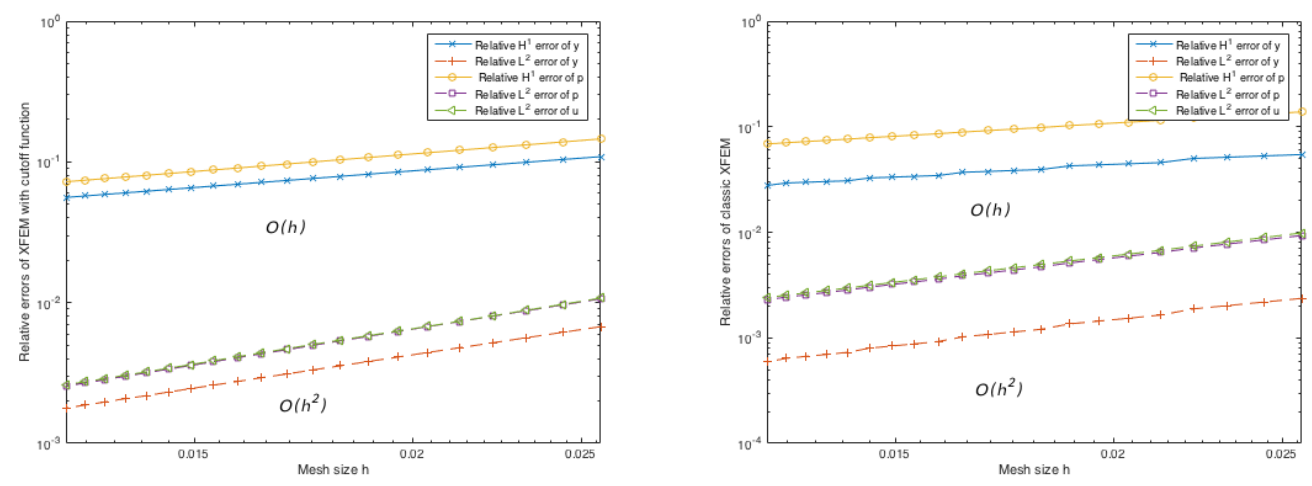

Figure 5: Convergence history of the cut XFEM (left) and classic XFEM (right) for Example 6.2.

Example 6.3. A constrained problem in a non-convex domain.

Let $\Omega$ be a $\frac{3}{4}$ unit circle (Figure 6 ), and take $\alpha=0.01$. We consider the optimal control problem (1.1) subject to

$$
\begin{cases}-\Delta y+y=u+f, & \text { in } \Omega, \\ y=0, & \text { on } \partial \Omega\end{cases}
$$


with the control constraint

$$
-0.3 \leq u \leq 1 \text {, a.e. on } \Omega \text {. }
$$

Set $r_{s}=0.5$, and let $\chi(r)$ be a polynomial with $r_{0}=0.01, r_{1}=0.99$, and let

$$
\begin{aligned}
& y=\left(r^{\frac{3}{2}}-r^{\frac{5}{2}}\right) \sin (\lambda \theta), \\
& p=\alpha\left(r^{\frac{3}{2}}-r^{\frac{5}{2}}\right) \sin (\lambda \theta), \\
& u=\min \left\{1, \max \left\{-\frac{p}{\alpha},-0.3\right\}\right\}
\end{aligned}
$$

be the analytical state, co-state and control, respectively. We note that the co-state $p$ satisfies

$$
\begin{cases}-\Delta p+p=y-y_{d} & \text { in } \Omega, \\ p=0, & \text { on } \partial \Omega .\end{cases}
$$

We apply the MATLAB mesh generator distmesh2d ([30]) to generate quasi-uniform triangular meshes (Figure 6): for $h=1 / 4,1 / 8,1 / 12,1 / 16, \cdots$,

$f d=@(q)\left(\operatorname{sqrt}\left(\operatorname{sum}\left(q^{2}, 2\right)\right)-1\right)+1 *(q(:, 1)>0+\operatorname{eps} \& q(:, 2)<0-$ eps $)$

$[q, t]=$ distmesh $2 d\left(f d, @\right.$ huniform, $\left.h,[-1,-1 ; 1,1 ;],\left[(0: h: 1)^{\prime},(0: h: 1)^{\prime} * 0 ;(0: h: 1)^{\prime} * 0,(-1: h: 0)^{\prime} ;\right]\right)$;

Tables 6-7 show results of the relative errors between $\left(y_{h}, p_{h}, u_{h}\right)$ and $(y, p, u)$, and Figure 5 shows the relative errors against the number of the mesh nodes, $N D$. It is known that the optimal convergence orders of the errors (against $N D$ ) in $H^{1}$ semi-norm and $L^{2}$ norm are $1 / 2,1$ respectively. We can see that the proposed methods yield optimal convergence rates. We note that in [2], graded meshes and a post-processing procedure were used to acquire optimal convergence for the $P_{1}$ element.

In Figures 8-10, we also show the cut XFEM solutions of the state, control and boundary of the active set at the mesh with $h=1 / 8$.
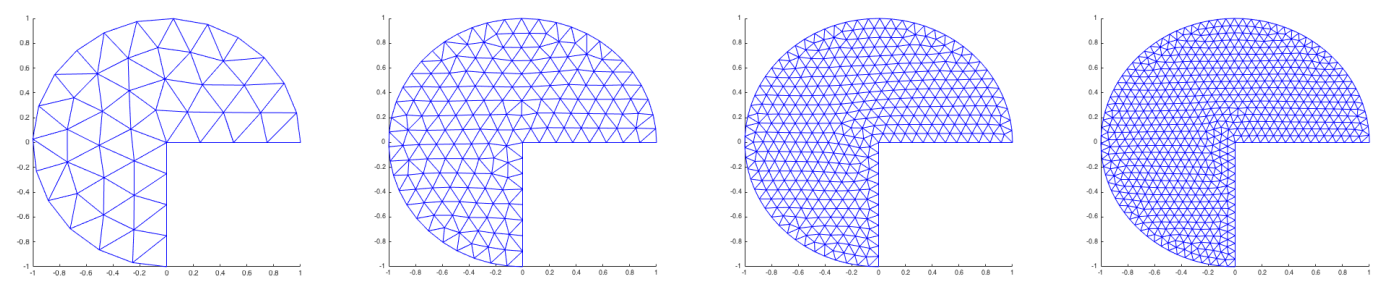

Figure 6: Domain and meshes for Example 3: $h=1 / 4(N D=51), 1 / 8(N D=186), 1 / 12(N D=$ $419), 1 / 16(N D=729)$ (left to right)

Table 6: Relative errors of the cut XFEM for Example 6.3.

\begin{tabular}{|c|c|c|c|c|c|c|c|c|c|c|}
\hline $1 / h$ & $\frac{\left|y-y_{h}\right|_{1}}{|y|_{1}}$ & order & $\frac{\left\|y-y_{h}\right\|_{0}}{\|y\|_{0}}$ & order & $\frac{\left|p-p_{h}\right|_{1}}{|p|_{1}}$ & order & $\frac{\left\|p-p_{h}\right\|_{0}}{\|p\|_{0}}$ & order & $\frac{\left\|u-u_{h}\right\|_{0}}{\|u\|_{0}}$ & order \\
\hline 4 & 0.1535 & & 0.0560 & & 0.2421 & & 0.2801 & & 0.2230 & \\
\hline 8 & 0.0776 & 0.53 & 0.0143 & 1.06 & 0.0934 & 0.74 & 0.0778 & 0.99 & 0.0553 & 1.08 \\
\hline 12 & 0.0506 & 0.53 & 0.0062 & 1.02 & 0.0555 & 0.64 & 0.0343 & 1.01 & 0.0239 & 1.03 \\
\hline 16 & 0.0383 & 0.50 & 0.0036 & 1.01 & 0.0403 & 0.58 & 0.0190 & 1.06 & 0.0133 & 1.05 \\
\hline
\end{tabular}

Table 7: Relative errors of the classic XFEM for Example 6.3.

\begin{tabular}{|c|c|c|c|c|c|c|c|c|c|c|}
\hline $1 / h$ & $\frac{\left|y-y_{h}\right|_{1}}{|y|_{1}}$ & order & $\frac{\left\|y-y_{h}\right\|_{0}}{\|y\|_{0}}$ & order & $\frac{\left|p-p_{h}\right|_{1}}{|p|_{1}}$ & order & $\frac{\left\|p-p_{h}\right\|_{0}}{\|p\|_{0}}$ & order & $\frac{\left\|u-u_{h}\right\|_{0}}{\|u\|_{0}}$ & order \\
\hline 4 & 0.0965 & & 0.0388 & & 0.1628 & & 0.1758 & & 0.1368 & \\
\hline 8 & 0.0567 & 0.41 & 0.0100 & 1.05 & 0.0657 & 0.70 & 0.0471 & 1.02 & 0.0359 & 1.03 \\
\hline 12 & 0.0378 & 0.50 & 0.0044 & 1.01 & 0.0410 & 0.58 & 0.0228 & 0.89 & 0.0165 & 0.96 \\
\hline 16 & 0.0287 & 0.49 & 0.0025 & 1.03 & 0.0302 & 0.55 & 0.0133 & 0.98 & 0.0094 & 1.01 \\
\hline
\end{tabular}



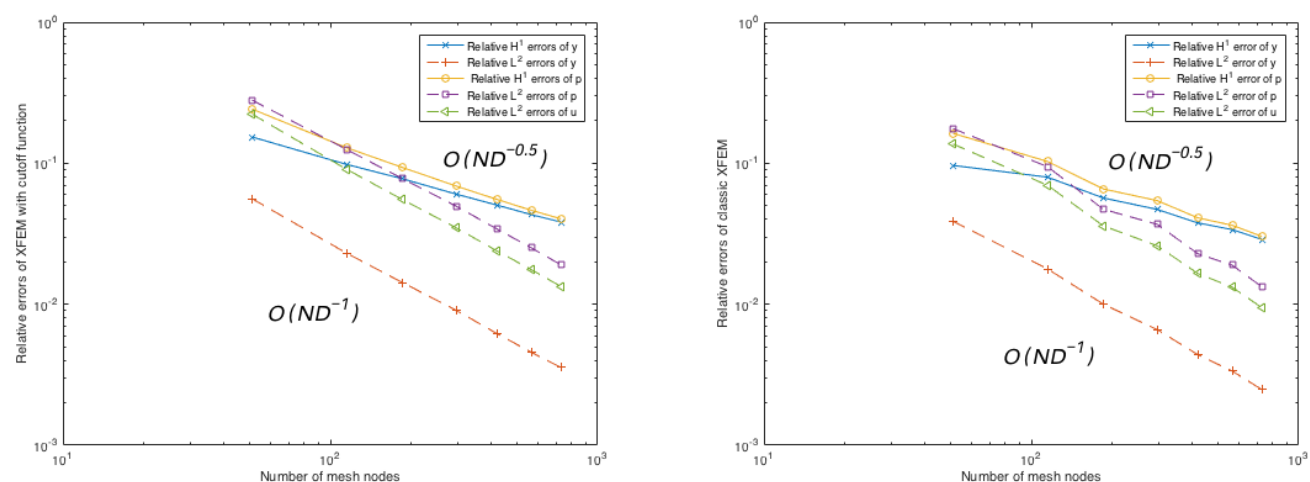

Figure 7: Convergence history of the cut XFEM (left) and classic XFEM (right) for Example 6.3.
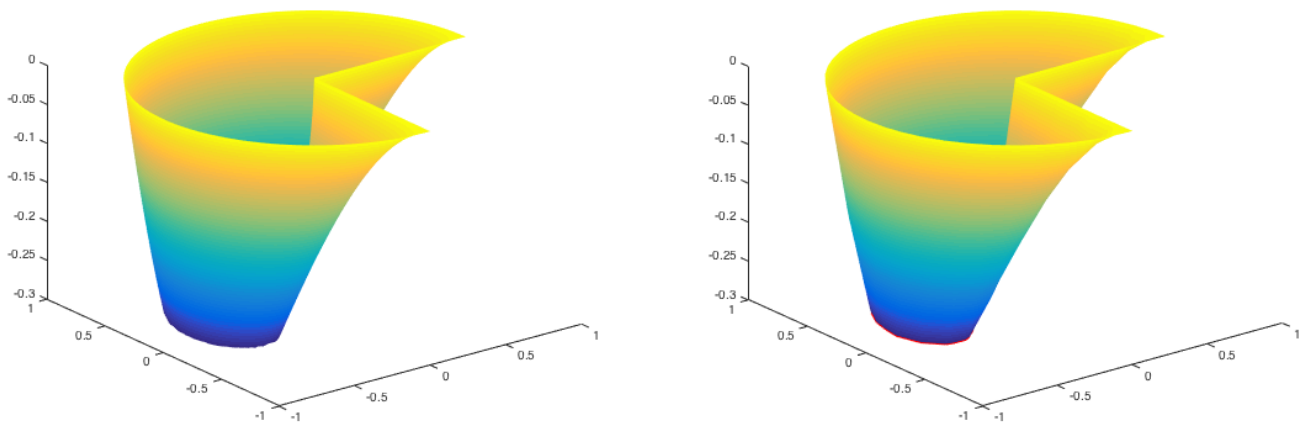

Figure 8: The exact control $u$ (left) and the discrete control $u_{h}$ by the cut XFEM (right) for Example 6.3 .
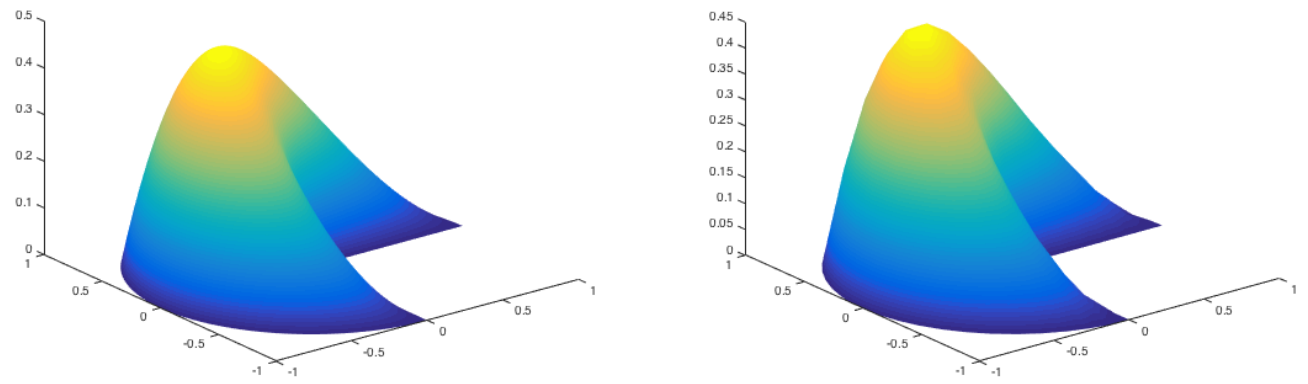

Figure 9: The exact state $y$ (left) and the discrete state $y_{h}$ by the cut XFEM for Example 6.3. 

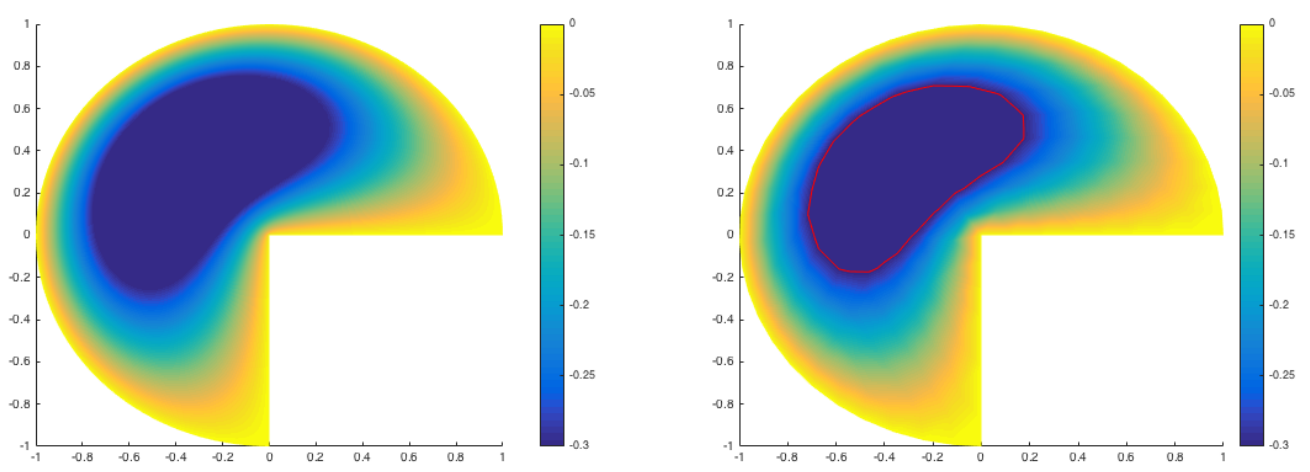

Figure 10: The active and inactive sets of the exact control $u$ (left) and the sets of the discrete control $u_{h}$ by the cut XFEM with $h=1 / 8$ : the red line is the boundary of active set for Example 6.3 .

\section{References}

[1] Thomas Apel, Johannes Pfefferer, and Arnd Sch. Finite element error estimates for neumann boundary control problems on graded meshes. Computational Optimization \& Applications, 52(1):3-28, 2012. 1

[2] Thomas Apel, Arnd Rosch, and Gunter Winkler. Optimal control in non-convex domains: a priori discretization error estimates. Calcolo, 44(3):137-158, 2007. 1, 13

[3] Thomas Apel, Anna-Margarete Sändig, and John R. Whiteman. Graded mesh refinement and error estimates for finite element solutions of elliptic boundary value problems in non-smooth domains. Mathematical Methods in the Applied Sciences, 19(1):63-85, 1996. 1

[4] Thomas Apel and Gunter Winkler. Optimal control under reduced regularity. Applied Numerical Mathematics, 59(9):2050-2064, 2009. 1

[5] Ivo Babuska, Uday Banerjee, and Kenan Kergrene. Strongly stable generalized finite element method: Application to interface problems. Computer Methods in Applied Mechanics \& Engineering, 2017. 2

[6] Ivo Babuska, R B Kellogg, and Juhani Pitkaranta. Direct and inverse error estimates for finite elements with mesh refinements. Numerische Mathematik, 33(4):447-471, 1979. 1

[7] Ivo Babuska and Robert Lipton. Optimal local approximation spaces for generalized finite element methods with application to multiscale problems. Siam Journal on Multiscale Modeling ES Simulation, 9(1):373-406, 2010. 2

[8] Ivo Babuska and Jens Markus Melenk. The partition of unity method. International Journal for Numerical Methods in Engineering, 40(4):727-758, 1997. 2

[9] Ivo Babuska and Michael B Rosenzweig. A finite element scheme for domains with corners. Numerische Mathematik, 20(1):1-21, 1972. 1, 10

[10] Roland Becker, Hartmut Kapp, and Rolf Rannacher. Finite element methods for optimal control of partial differential equations: Basic concept. Siam Journal on Control and Optimization, 39(1):113132, 2000. 1

[11] Ted Belytschko and T Black. Elastic crack growth in finite elements with minimal remeshing. International Journal for Numerical Methods in Engineering, 45(5):601-620, 1999. 2, 5

[12] Ted Belytschko, Robert Gracie, and Giulio Ventura. A review of extended/generalized finite element methods for material modeling. Modelling and Simulation in Materials Science and Engineering, 17(4):043001, 2009. 2 
[13] Olaf Benedix and Boris Vexler. A posteriori error estimation and adaptivity for elliptic optimal control problems with state constraints. Computational Optimization and Applications, 44(1):3-25, 2009. 1

[14] Stefano Berrone, Sandra Pieraccini, and Stefano Scialo. On simulations of discrete fracture network flows with an optimization-based extended finite element method. Siam Journal on Scientific Computing, 35(2):A908-A935, 2013. 2

[15] Heribert Blum and Manfred Dobrowolski. On finite element methods for elliptic equations on domains with corners. Computing, 28(1):53-63, 1982. 1, 2

[16] Eduardo Casas. Control of an elliptic problem with pointwise state constraints. Siam Journal on Control and Optimization, 24(6):1309-1318, 1986. 1

[17] Richard S Falk. Approximation of a class of optimal control problems with order of convergence estimates. Journal of Mathematical Analysis and Applications, 44(1):28-47, 1973. 1

[18] Wei Gong and Ningning Yan. Adaptive finite element method for elliptic optimal control problems: convergence and optimality. Numerische Mathematik, 135(4):1121-1170, 2017. 1

[19] Pierre Grisvard. Elliptic problems in nonsmooth domains. Pitman Advanced Pub. Program, 1985.2

[20] Michael Hintermuller and Ronald H W Hoppe. Goal-oriented adaptivity in pointwise state constrained optimal control of partial differential equations. Siam Journal on Control and Optimization, 48(8):5468-5487, 2010. 1

[21] Michael Hinze. A variational discretization concept in control constrained optimization: the linearquadratic case. Computational Optimization and Applications, 30(1):45-61, 2005. 1, 2, 6

[22] Michael Hinze and Morten Vierling. Variational discretization and semi-smooth newton methods; implementation, convergence and globalization in pde constrained optimization with control constraints. Immunogenetics, 55(7):429-436, 2009. 1, 2, 8

[23] Patrick Ciarlet Jr and Jiwen He. The singular complement method for $2 \mathrm{~d}$ scalar problems. Comptes Rendus Mathematique, 336(4):353-358, 2003. 1, 2

[24] Kenan Kergrene, Ivo Babuska, and Uday Banerjee. Stable generalized finite element method and associated iterative schemes; application to interface problems. Computer Methods in Applied Mechanics \& Engineering, 305:1-36, 2016. 2

[25] Ruo Li, Wenbin Liu, Heping Ma, and Tao Tang. Adaptive finite element approximation for distributed elliptic optimal control problems. Siam Journal on Control and Optimization, 41(5):13211349, 2002. 1

[26] Christian Meyer and Arnd Rosch. Superconvergence properties of optimal control problems. Siam Journal on Control and Optimization, 43(3):970-985, 2004. 1

[27] Nicolas Moes, John E Dolbow, and Ted Belytschko. A finite element method for crack growth without remeshing. International Journal for Numerical Methods in Engineering, 46(1):131-150, 1999. 2

[28] Serge Nicaise, Yves Renard, and Elie Chahine. Optimal convergence analysis for the extended finite element method. International Journal for Numerical Methods in Engineering, 86(4-5):528-548, 2011. 5

[29] L. A. Oganesyan and L. A. Rukhovets. Variational-difference schemes for linear second-order elliptic equations in a two-dimensional region with piecewise smooth boundary. Ussr Computational Mathematics $\&$ Mathematical Physics, 8(1):129,138,142-135,139,152, 1968. 1

[30] Per Olof Persson and Gilbert Strang. A simple mesh generator in matlab. Siam Review, 46(2):329345, 2004. 13 
[31] Arnd Rosch, Kunibert G Siebert, and S Steinig. Reliable a posteriori error estimation for stateconstrained optimal control. Computational Optimization and Applications, 68(1):121-162, 2017. 1

[32] Arnd Rosch and Simeon Steinig. A priori error estimates for a state-constrained elliptic optimal control problem. Mathematical Modelling and Numerical Analysis, 46(5):1107-1120, 2012. 1

[33] Arnd Rosch and Daniel Wachsmuth. A-posteriori error estimates for optimal control problems with state and control constraints. Numerische Mathematik, 120(4):733-762, 2012. 1

[34] A. H. Schatz and L. B. Wahlbin. Maximum norm estimates in the finite element method on plane polygonal domains. Mathematics of Computation, 33(146):465-492, 1979. 1

[35] Rene Schneider and Gerd Wachsmuth. A posteriori error estimation for control-constrained, linearquadratic optimal control problems. SIAM Journal on Numerical Analysis, 54(2):1169-1192, 2016. 1

[36] David Sevilla and Daniel Wachsmuth. Polynomial integration on regions defined by a triangle and a conic. In International Symposium on Symbolic and Algebraic Computation, pages 163-170, 2010. 9

[37] Yongxing Shen and Adrian Lew. Stability and convergence proofs for a discontinuous-galerkin-based extended finite element method for fracture mechanics. Computer Methods in Applied Mechanics 83 Engineering, 199(37):2360-2382, 2010. 2, 3

[38] Soheil Soghrati and Philippe H. Geubelle. A 3d interface-enriched generalized finite element method for weakly discontinuous problems with complex internal geometries. Computer Methods in Applied Mechanics \& Engineering, 217-220(1):46-57, 2012. 2

[39] Gilbert Strang and George J Fix. An analysis of the finite element method. Prentice-Hall, 1973. 1

[40] T Strouboulis, Ivo Babuska, and K Copps. The design and analysis of the generalized finite element method. Computer Methods in Applied Mechanics and Engineering, 181:43-69, 2000. 2

[41] Theofanis Strouboulis, Ivo Babuska, and Realino Hidajat. The generalized finite element method for Helmholtz equation: Theory, computation, and open problems. Computer Methods in Applied Mechanics \& Engineering, 195(37-40):4711-4731, 2006. 2

[42] Dan Tiba and Fredi Troltzsch. Error estimates for the discretization of state constrained convex control problems. Numerical Functional Analysis and Optimization, 17:1005-1028, 1996. 1

[43] Fredi Troltzsch. Optimal control of partial differential equations: Theory, methods and applications. Siam Journal on Control \&J Optimization, 112(2):399, 2010. 3

[44] Chao Chao Yang, Tao Wang, and Xiaoping Xie. An interface-unfitted finite element method for elliptic interface optimal control problem. arXiv:1805.04844v2, 2018. 1 